\title{
Antithrombotic and Anti-Inflammatory Effects of Fondaparinux and Enoxaparin in Hospitalized COVID-19 Patients: The FONDENOXAVID Study
}

This article was published in the following Dove Press journal:

Journal of Blood Medicine

\section{Giuseppe Cardillo' Giuseppe Vito Viggiano ${ }^{2}$ \\ Vincenzo Russo ${ }^{3}$ Sara Mangiacapra ${ }^{4}$ \\ Antonella Cavalli ${ }^{5}$ \\ Giampiero Castaldo ${ }^{2}$ \\ Federica Agrusta ${ }^{2}$ \\ Annamaria Bellizzi Snr 5 \\ Maria Amitrano Snr ${ }^{4}$ \\ Mariateresa lannuzzo ${ }^{6}$ \\ Clara Sacco ${ }^{7}$ \\ Corrado Lodigiani (iD) ${ }^{7}$ \\ Andrea Fontanella (D) ${ }^{6}$ \\ Pierpaolo Di Micco ${ }^{6}$ \\ For The FondenoxavidStudy \\ Group \\ 'Medylab, Biochimica Avanzata Laboratory, Naples, Italy; ${ }^{2} \mathrm{UO}$ Pronto \\ Soccorso e Medicina D'urgenza, Semintensiva Covid, Ospedale Ramazzini \\ Di Carpi - AUSL Modena, Modena, Italy; ${ }^{3}$ Department of Translational \\ Medical Sciences, University of Campania Luigi Vanvitelli Monaldi Hospital, Piazzale Ettore Ruggeri, Naples 80 I3I, Italy; ${ }^{4}$ UOC Medicina, AO Moscati, Avellino, Italy; ${ }^{5}$ UOC Medicina-Covid, Presidio Opsedaliero Frangipane Di Arianolrpino (AV), Naples, Italy; ${ }^{6}$ UOC Medicina, Ospedale Fatebenefratelli di Napoli, Naples, Italy; ${ }^{7}$ Humanitas Clinical and Research Center IRCCS, Rozzano, Italy}

Correspondence: Pierpaolo Di Micco Email pdimicco@libero.it
Background: Since the outbreak of novel coronavirus SARS-CoV2 around the world, great attention has been paid to the effects of such antithrombotic drugs as heparinoids, because they have antiviral action in vitro and antithrombotic actions in vivo. We conducted a retrospective analysis in inpatients with confirmed COVID-19 on the anti-inflammatory and antithrombotic effects of enoxaparin and fondaparinux at prophylactic doses.

Methods: This retrospective cohort study used patients with confirmed COVID-19 during the first months of the Italian outbreak from February 18 to April 30, 2020. Our aim was to compare clinical characteristics, prophylactic treatment, markers of inflammation, and thrombotic outcomes in inpatients positive for SARS-CoV2 during hospitalization associated with thromboprophylaxis with enoxaparin (40 $\mathrm{mg}$ or $60 \mathrm{mg}$ once daily) or fondaparinux ( $2.5 \mathrm{mg}$ once daily). Statistical analysis was conducted with using MatLab R2016B and ad hoc functions.

Results: There were no significatant differences in clinical characteristics between patients that used enoxaparin or fondaparinux as thromboprophylaxis for SARS-CoV2. No differences were found in D-dimer and fibrinogen levels either, which were used as markers of inflammation during the infection at testing on admission and after 3 weeks.Significant differences in CRP, IL6, and LDH were found in patients after 21 days' treatment.

Discussion: Increased levels of fibrinogen and D-dimer in patients with confirmed COVID19 have been reported in several studies. Our results showed that anti-inflammatory effects of fondaparinux and enoxaparin after 3 weeks of prophylactic treatment were similar when levels of fibrinogen and D-dimer were considered. Furthermore, levels of CRP showed a decrease in patients treated with enoxaparin and fondaparinux, although the decrease in the fondaparinux group seems to be more relevant.

Keywords: venous thromboembolism, fibrinogen, D-dimer, COVID-19, SARS-CoV2, fondaparinux, enoxaparin

\section{Introduction}

Following the COVID-19 outbreak, ${ }^{1}$ which has spread rapidly from China to other countries in the world with alarming morbidity and mortality, ${ }^{2}$ several therapeutic strategies have been reported, including oxygen support ${ }^{3}$ and experimental antiviral therapies. $^{4}$ Based on the coexistence of inflammation and hypercoagulable state ${ }^{5}$ leading to increased risk of venous thromboembolism events (VTEs), ${ }^{6-8}$ the use of low-molecular weight heparins (LMWHs) is recommended as part of standard 
therapy in hospitalized COVID-19 patients. ${ }^{9,10}$ LMWHs, such as enoxaparin, seem to act with a specific antithrombotic action and also with additional anti-inflammatory and antiviral activities in vitro against SARS-CoV2. ${ }^{11,12}$

Despite the antithrombotic role of enoxaparin and also fondaparinux in preventing VTEs in COVID-19 patients,${ }^{13-15}$ few data are available on their antithrombotic and anti-inflammatory effects in vivo. The aim of our study was to evaluate the antithrombotic and anti-inflammatory effects of fondaparinux and enoxaparin among COVID-19 patients according to selected laboratory markers found in the FONDENOXAVID study.

\section{Methods}

\section{FONDENOXAVID Study}

\section{Inclusion Criteria}

Clinical data of selected patients with symptomatic, confirmed COVID-19 were analyzed by a retrospective analysis in order to understand incidence and mortality for VTEs and also to understand trends of inflammatory and clotting markers.

Selected clinical records of these patients were chosen according to their thromboprophylaxis with fondaparinux or enoxaparin at doses suggested by international guidelines for VTE prevention.

Oral or written informed consent were arranged in agreement with the Ethical Committee of the University of Campania and in accordance with the Declaration of Helsinki and given to each patient for participation in this retrospective analysis - FONDENOXAVID. The ethical committee approved the registry on anticoagulant treatments $(751 / 2019)$.

Physicians participating in the FONDENOXAVID retrospective analysis made all efforts to select consecutive patients.

Data were recorded onto a computer-based report at each participating hospital and sent to a centralized coordinating center through a secure system.

Several Italian Hospitals agreed to perform this analysis: Ospedale Ramazzini di Carpi, Italy; Moscati Hospital, Avellino, Italy, Ospedale Fatebenefratelli di Napoli, Italy, Thrombosis Center, IRCCS Humanitas Clinical and Research Center, Rozzano, Italy, and Presidio Ospedaliero Frangipane di Ariano Irpino, Italy.

All hospital provided clinical data derived from records from specialistic nonintensive and subintensive COVID wards.
FONDENOXAVID also used electronic data monitoring to detect inconsistencies or errors, and attempted to resolve discrepancies by contacting the local coordinators.

\section{Exclusion Criteria}

Patients aged $<18$ years were excluded from the retrospective analysis, as were those anticoagulants at therapeutic doses before SARS-CoV2 diagnosis for any medical reason patients positive for SARS-CoV2, but with recent bleeding (ie, within 30 days of hospital admission).

Patients were also excluded if they were currently participating in any clinical trials regarding COVID-19 or VTEs.

\section{Design}

This retrospective cohort study used patients with SARSCoV2 during the Italian outbreak from February 18 to April 30, 2020.

Our aim was to compare clinical characteristics, laboratory markers, prophylactic treatment, and rate of VTEs in inpatients positive for SARS-CoV2 on primary thromboprophylaxis with enoxaparin (40 $\mathrm{mg}$ or $60 \mathrm{mg}$ once daily) or fondaparinux ( $2.5 \mathrm{mg}$ once daily).

Inflammatory markers to detect progression of prothrombotic state were recorded: levels of fibrinogen, Ddimer, LDH, IL6, and CRP were sequentially tested. In particular, D-dimer testing was performed at admission, day 21 after admission, or in cases of worsening of lung performance. In order to monitor lung performance in COVID-19, SIRS criteria were adopted. ${ }^{16}$

Main outcomes were rate of VTEs (ie, deep-vein thrombosis DVT, superficial VT, pulmonary embolism), mortality for VTEs, and levels of fibrinogen, IL6, CRP, $\mathrm{LDH}$, and D-dimer as markers of inflammatory and prothrombotic state during the disease.

\section{Study Population}

In total, 100 consecutive symptomatic patients with laboratory-proven COVID-19 admitted to internal medicine units of five Italian hospitals from February 18 to April 30, 2020 were selected for this retrospective analysis. All selected COVID-19 patients were receiving VTE prophylaxis according to the current international guidelines. ${ }^{9}$

The institutional ethical committee (FBGD-90320) approved the study, and patients gave standard written consent to the use of their data. 
Table I Demographic, clinical, and laboratory characteristics of the study population

\begin{tabular}{|l|c|c|c|}
\hline & Enoxaparin, $\mathbf{n = 6 2}$ & Fondaparinux, $\mathbf{n = 3 8}$ & $\mathbf{p}$-value \\
\hline Male, $\mathrm{n}(\%)$ & $40(65 \%)$ & $23(61 \%)$ & 0.76 \\
\hline Age $<40$ years, $\mathrm{n}(\%)$ & $3(5 \%)$ & $6(16 \%)$ & 0.09 \\
\hline Age $40-60$ years, $\mathrm{n}(\%)$ & $34(34 \%)$ & $17(45 \%)$ \\
\hline Age $\geq 60$ years, $\mathrm{n}(\%)$ & $38(61 \%)$ & $15(39 \%)$ & $2(5 \%)$ \\
\hline VT & $5(8 \%)$ & 0 & 0.76 \\
\hline PE & $4(6 \%)$ & $4(10 \%)$ & 0.12 \\
\hline Deaths & $6(9 \%)$ & \\
\hline
\end{tabular}

Note: All variables were analyzed with Barnard's test, except age (Fisher's test).

Abbreviations: VT, vein thrombosis; PE, pulmonary embolism.

Serum levels of fibrinogen, D-dimer, LDH, IL6, and CRP were evaluated at admission and after 3 weeks to evaluate changes in inflammatory and prothrombotic clotting markers during treatment.

All VTEs of the lower limbs with or without associated pulmonary embolism diagnosed with objective methods were recorded.

\section{Statistical Analysis}

The Anderson-Darling test was used to analyze data normality. Continuous variables are reported using median and IQRs. Categorical variables are expressed as frequency counts and percentages. Differences between unpaired data were evaluated using two-tailed FlignerPolicello and Fligner-Killeen tests and paired data using the one-tailed Wilcoxon test and Hodges-Lehmann estimator of mean difference. Differences among three groups were evaluated using the two-tailed Dunn multiple-comparison test with Bonferroni correction. Categorical data

Table 2 Classes of drugs used in the described cohort of patients treated for SARS-CoV2

\begin{tabular}{|l|c|}
\hline Patients (n=100) & $\mathbf{n}$ \\
\hline Antibiotics & 64 \\
Biologics (tocilizumab or others) & 12 \\
Antivirals & 21 \\
Steroids & 55 \\
Immunomodulants (hydroxychloroquine or others) & 65 \\
Heparinoids (enoxaparin or fondaparinux) & 100 \\
NSAIDs, daily use & 20 \\
Acetaminophen & 68 \\
\hline
\end{tabular}

Abbreviations: NSAIDs, nonsteroidal anti-inflammatory drugs. were evaluated using Barnard's or Fisher's test. Statistical comparisons were performed using MatLab R2018a.

\section{Results}

Baseline clinical and laboratory characteristics of the study population are shown in Table 1. No significant differences between enoxaparin and fondaparinux group has been shown.

Table 2 reports all categories of drug used to treat SARS-CoV2 in our cohort, in which heparinoids (ie, enoxaparin and fondaparinux) were the only drugs to reach $100 \%$ of patients.

In Table 3, all VTEs that we detected with objective methods during the analysis are reported. VT of the lower limbs was detected in five patients in the enoxaparin group vs two patients in the fondaparinux group: three DVT, one isolated distal VT, and one superficial VT for the enoxaparin group and two DVTs for the fondaparinux group. Pulmonary embolism was detected in 4 patients in the enoxaparin group while no pulmonary embolism was found in the fondaparinux group. Three events of proximal symptomatic DVT were found in the group on enoxaparin, and two events of symptomatic proximal DVT were found in the groupon fondaparinux. Furthermore two asymptomatic VTs were found in the patients on enoxaparin: one superficial VT of the lower limbs and one isolated distal DVT. In the enoxaparin group, four thrombotic events were associated with pulmonary embolisms. pPulmonary embolisms were detected with lung CT scans. Data on distribution of thrombotic eventsaere summarized in Tables 1 and 3. No statistical differences were found between groups.

In Table 4, differences in of D-dimer, fibrinogen, CRP, and IL6 levels at hospital admission and after 3 weeks of treatment are reported. 
Table 3 Different doses of enoxaparin and fondaparinux in patients with COVID-19 at baseline with occurrence of vein-thrombosis events (VTEs)

\begin{tabular}{|l|c|c|c|}
\hline & $\mathbf{n}$ & Type of VTE & Clinical signs of suspected VTE \\
\hline $\begin{array}{l}\text { Enoxaparin twice daily after VTE } \\
\text { diagnosis }\end{array}$ & 5 & $\begin{array}{c}\text { Three proximal DVT, one IDVT, one SVT, four f } \\
\text { them with PE }\end{array}$ & $\begin{array}{c}\text { Twosymptomatic DVT, two asymptomatic } \\
\text { IDVT and SVT }\end{array}$ \\
\hline $\begin{array}{l}\text { Fondaparinux therapeutic dosage after } \\
\text { VTE diagnosis }\end{array}$ & 2 & 2 proximal DVT & Two symptomatic DVT \\
\hline
\end{tabular}

Abbreviations: DVT, deep-vein thrombosis; IDVT, isolated DVT; SVT, superficial VT; PE, pulmonary embolism.

Table 4 Distributions of inflammatory markers between enoxaparin and fondaparinux groups at baseline and after 3 weeks

\begin{tabular}{|c|c|c|c|c|}
\hline D-dimer, $\mu g / d L$ & Enoxaparin, $n=62$ & Fondaparinux, $n=38$ & FP test $p$-value & FK test $p$-value \\
\hline Admission & $710.5(520-1,208)$ & $643.5(502-919)$ & 0.0972 & 0.1362 \\
\hline 3 weeks later & $602(428-1,230)$ & $606(450-810)$ & 0.3783 & 0.2059 \\
\hline HL mean difference & $-151(-292.5$ to 16.5$)$ & $-42.75(-210.75$ to 133$)$ & & \\
\hline Wilcoxon test $p$-value & 0.015217 & 0.21036 & & \\
\hline \multicolumn{5}{|l|}{ Fibrinogen, $\mathrm{mg} / \mathrm{dL}$} \\
\hline Admission & $600(478-734.5)$ & $569.5(503-632)$ & 0.1501 & 0.0758 \\
\hline 3 weeks later & $631(497-722.5)$ & $535(450-630)$ & 0.0114 & 0.0695 \\
\hline HL mean difference & 14.8 (-37.0 to 59.5$)$ & $-16.5(-67$ to 38$)$ & & \\
\hline Wilcoxon test $p$-value & 0.28607 & 0.28219 & & \\
\hline \multicolumn{5}{|l|}{ CRP, mg/dL } \\
\hline Admission & II.5 (3.6-22) & $44(15-52)$ & 0.000004 & 0.0011 \\
\hline 3 weeks later & $13(5-40.5)$ & $15(9-2 \mid)$ & 0.3051 & 0.1652 \\
\hline HL mean difference & $6(0.5-13.8)$ & $-22.5(-34.5$ to 11$)$ & & \\
\hline Wilcoxon test $p$-value & 0.0063151 & 0.00050565 & & \\
\hline \multicolumn{5}{|l|}{ LDH, U/L } \\
\hline Admission & $252.5(209-343)$ & 301 (232-349) & 0.2085 & 0.7407 \\
\hline 3 weeks later & $212.5(202-255)$ & $239(203-264)$ & 0.0889 & 0.2446 \\
\hline HL mean difference & $-62(-88.5$ to 38$)$ & $-55(-140$ to 4$)$ & & \\
\hline Wilcoxon test $p$-value & 0.0000002 & 0.0128 & & \\
\hline \multicolumn{5}{|l|}{ IL6, pg/mL } \\
\hline Admission & $16(13.2-20)$ & $15(13-20)$ & 0.4891 & 0.9692 \\
\hline 3 weeks later & $6(4-9.8)$ & $6(4-9.8)$ & 0.4946 & 0.9692 \\
\hline $\mathrm{HL}$ mean difference & $-9(-11.0$ to 6.5$)$ & $-9(-11.0$ to 6.5$)$ & & \\
\hline Wilcoxon test $p$-value & 0.0000002 & 0.00007 & & \\
\hline
\end{tabular}

Abbreviations: FP, Fligner-Policello; FK, Fligner-Killeen; HL, Hodges-Lehmann. 
No differences in medians or IQRs for unmatched D-dimer were found. Hodges-Lehmann mean-difference estimators were not significant (95\% CIs including 0$)$ for fondaparinux or enoxaparin, and the one-tailed Wilcoxon test showed the same results. We can conclude that fondaparinux is not effective in reducing D-dimer, but enoxaparin is effective.

No differences in medians or IQRs on unmatched fibrinogen distributions were found. Hodges-Lehmann mean-difference estimators were not significant ( $95 \%$ CIs including 0 ), and the one-tailed Wilcoxon test did not revealdifferences between groups. As such, we can conclude that neither drug had a significant effect on fibrinogen.

Data on CRP distributions were found to be different: the fondaparinux group showed a higher median and IQR (maybe more severe cases were treated with fondaparinux) than the enoxaparin group. However, 3 weeks later, both groups had reached the same median and IQR. In the enoxaparin group, the Hodges-Lehmann estimator was $>0$ while in the fondaparinux group it was $<0$. We can conclude (from the IQR too) that enoxaparin is not effective in reducing CRP, while fondaparinux is effective.

No differences in medians or IQRs on unmatched LDH distributions were found Hodges-Lehmann mean-difference estimators and one-tailed Wilcoxon test results were found to be significant in both groups. As such, we can conclude that both drugs were effective in reducing LDH.

Finally, no differences in medians or IQRs for unmatched IL6 were found. Hodges-Lehmann mean-difference estimators and one-tailed Wilcoxon test results were found to be significant in both groups. Therefore, we can conclude that both drugs were effective in reducing IL6.

\section{Discussion}

Previous reports on the COVID-19 outbreak have focused on the prognostic roles of laboratory markers regarding their involvement in the pathophysiology of infectious or inflammatory disease. The prognostic roles of D-dimer, fibrinogen, CRP, LDH, IL6, and other tests during COVID-19 outbreak have been underlined. ${ }^{2,5}$

$\mathrm{D}$-dimer testing is one of the laboratory procedures used to investigate or exclude venous thromboembolism, ${ }^{17}$ and its levels may also have prognostic value after confirmation of a VTE. ${ }^{18}$ Usually, D-dimer ha negative predictive value, because an increase may also be present in other diseases, such as inflammations and/or infections.19,20 COVID-19 is in fact a specific infection that is associated with increased D-dimer levels per se.,
In the same way, increased fibrinogen levels have been associated with increased rates of VTEs and severe infections due to SARS-CoV2. ${ }^{4,5}$

On the other hand, CRP is one of the most used inflammatory markers to screen acute and chronic infections and inflammation, and LDH is one of the most common markers of chronic disease associated with cytolysis. Retrospective analysis of COVID-19 cohort has confirmed these roles. ${ }^{21}$

Also, IL6 levels have been associated with differing evolution of lung inflammation ${ }^{21,22}$ in patients with confirmed COVID-19.

The main objective and findings of our study were the reduction of inflammatory markers after thromboprophylaxis with enoxaparin or fondaparinux. The findings point to the ancillary properties of these drugs in vivo.

Anti-inflammatory effects of heparins have been reported in vitro and in vivo, and are based on their role as a coadjuvant of serpins. ${ }^{23}$ Reductions in inflammatory markers during administration of enoxaparin has also been reported in animal models. ${ }^{24}$ These mechanisms also have a role in reduction of IL6 levels during inflammation induced by SARS-CoV2. ${ }^{10,25-27}$

Standard treatment for COVID-19 in fact is based on the administration of several kind of drugs with multiple actions, in order to reduce inflammatory damage (ie, antivirals, immunomodulants, antibiotics, steroids, antithrombotics). Due to their pharmacological properties, heparinoids can fight SARSCoV2 in multiple ways: a specific antiviral action of heparin toward SARS-CoV2 has been found in vitro and in vivo, ${ }^{10,23-}$ ${ }^{27}$ as well a specific anti-inflammatory action of heparinoids and fondaparinux. ${ }^{28,29}$ Of course, these additional properties should be added to the well-known antithrombotic actions of both drugs. As the specific action of heparin is due to the pentasaccharide sequence present in heparins and fondaparinux, ${ }^{30}$ we postulated that all therapeutic actions exerted by enoxaparin could also be achieved by fondaparinux.

We found anti-inflammatory effects for enoxaparin and fondaparinux in levels of D-dimer, fibrinogen, CRP, LDH, and IL6 at admission and after 3weeks of treatment.

A progressive reduction in all values was recorded in both groups for D-dimer, fibrinogen, CRP, LDH, and IL6 levels.

Reduction in D-dimer levels after 3 weeks of prophylaxis with heparinoids was similar for patients treated with enoxaparin or fondaparinux, although enoxaparin seems to be more effective in reducing D-dimer levels.

Both drugs were also effective in reducing median LDH and IL6 levels. 
On the contrary, neither drug seemed to be effective for fibrinogen reduction, though the fondaparinux group showed a lower median.

As such, we can assume that the use of such heparinoids as enoxaparin or fondaparinux has multiple clinical advantages for patients with confirmed COVID-19, based on the reductions in inflammatory markers and VTE rate found.

Interestingly, as shown in Table 2, heparinoids were the only drugs used in all patients, so we can postulate that results from the clinical and laboratory points of view are also associated with their use. In particular, their antiinflammatory effects contributed to improvements in levels of inflammatory markers. Based on these data, we can speculate on specific actions of enoxaparin or fondaparinux on specific inflammatory markers, but this topic would be better investigated on a large population and include a composite clinical evaluation (eg, clinical performance, laboratory evaluation, and specific outcomes). We also emphasize that prolonged administration of enoxaparin or fondaparinux in inpatients with COVID-19 may be associated with ancillary anti-inflammatory responses to the known antithrombotic action of these drugs.

We found that the incidence of VTEs in our cohort was lower than other studies (nearly 10\%), although abnormal and increased values of $\mathrm{D}$-dimer were present in $>75 \%$ of patients. This could be related to the difficulty in investigating DVT in patients with COVID-19. ${ }^{31}$ In this clinical setting, the incidence of thrombotic events was similar in both groups, confirming that both pharmacological approaches were valid in terms of support for these patients.

Therefore, in this specific field we have added to and enlarged the knowledge base concerning the use of enoxaparin and fondaparinux in this clinical setting. Studies have provided more data on the use of enoxaparin or other heparins than fondaparinux in VTE prevention of COVID-19 patients and reduction of anti-inflammatory markers.

\section{Study Limitations}

Our study has several limitations. First of all, of patient selection was retrospective. The number of patients that received thromboprophylaxis with fondaparinux $2.5 \mathrm{mg}$ daily in the hospitals involved was very low. Furthermore, the fondaparinux has a unique dosage for thromboprophylaxis, while different dosages are available for enoxaparin. We selected only patients that received the standard dosage of 4,000 U daily.
The small number of thrombotic events that we noted in the retrospective analysis could be related to the fact that ultrasound scans for VT were performed between days 5 and 8 of hospitalization. The best timing for ultrasound scans for VT in these patients is still a matter of discussion in daily clinical management.

Another limitation could be related to the minority of patients that developed VTEs, because in these patients heparinoid dose was changed. However, the number of patients with VTEs was very small, and statistical analysis did not reflect this limitation.

Last but not least, outcomes of events were limited to 4 weeks. More detailed and prolonged studies should improve on this first study in thise field.

\section{Conclusion}

This retrospective analysis on inpatients with confirmed COVID-19 was based on the ancillary properties of the heparinoids enoxaparin and fondaparinux, which have anti-inflammatory effects in vitro and in vivo and antithrombotic effects.

We performed a combined analysis on the incidence of VTEs confirmed by objective methods and anti-inflammatory actions in patients treated with prophylactic doses of enoxaparin or fondaparinux.

Both drugs were able to exert anti-inflammatory effects, as shown by reduction in all markers. Although fondaparinux showed little advantage in reducing CRP levels, enoxaparin did with regard to D-dimer levels. These additional aspects that we found in our analysis should always be joined to clinical aspects.

\section{Funding}

No funding was received for this manuscript.

\section{Disclosure}

The authors certify that they have no conflicts of interest to declare for this report.

\section{References}

1. Sohrabi C, Alsafi Z, O'Neill N, et al. World Health Organization declares global emergency: a review of the 2019 novel coronavirus (COVID-19). Int J Surg. 2020;76:71-76. doi:10.1016/j.ijsu.2020.02.034

2. Juul S, Nielsen N, Bentzer P, et al. Interventions for treatment of COVID-19: a protocol for a living systematic review with network meta-analysis including individual patient data (The LIVING Project). Syst Rev. 2020;9(1):108. doi:10.1186/s13643-020-01371-0

3. Cao B, Wang Y, Wen D, et al. A trial of Lopinavir-Ritonavir in adults hospitalized with severe Covid-19. N Engl J Med. 2020;382(19):17871799. doi:10.1056/NEJMoa2001282 
4. Di Micco P, Russo V, Carannante N, et al. Clotting factors in COVID-19: epidemiological association and prognostic values in different clinical presentations in an Italian cohort. J Clin Med. 2020;9(5):1371. doi:10.3390/jcm9051371

5. Tang N, Li D, Wang X, Sun Z. Abnormal coagulation parameters are associated with poor prognosis in patients with novel coronavirus pneumonia. J Thromb Haemost. 2020;18(4):844-847. doi:10.1111/ jth. 14768

6. Scudiero F, Silverio A, Di Maio M, et al. Cov-IT network. Pulmonary embolism in COVID-19 patients: prevalence, predictors and clinical outcome. Thromb Res. 2021;198:34-39. doi:10.1016/j.thromres.2020.11.017

7. Klok FA, Kruip MJHA, van der Meer NJM, et al. Incidence of thrombotic complications in critically ill ICU patients with COVID19. Thromb Res. 2020; 191:145-7.

8. Cattaneo M, Bertinato EM, Birocchi S, et al. Pulmonary embolism or pulmonary thrombosis in COVID-19? Is the recommendation to use high-dose heparin for thromboprophylaxis justified? Thromb Haemost. 2020.

9. Bikdeli B, Madhavan MV, Jimenez D, et al. COVID-19 and thrombotic or thromboembolic disease: implications for prevention, antithrombotic therapy, and follow-up. J Am Coll Cardiol. 2020;75 (23):2950-73.

10. Shastri MD, Stewart N, Horne J, et al. In-vitro suppression of IL-6 and IL- 8 release from human pulmonary epithelial cells by nonanticoagulant fraction of Enoxaparin. PLoS One. 2015;10(5): e0126763. doi:10.1371/journal.pone.0126763

11. Di Micco P, Di Micco G, Russo V, et al. Blood targets of adjuvant drugs against COVID19. J Blood Med. 2020;11:237-241. doi:10.2147/JBM.S256121

12. Tiwari V, Beer JC, Sankaranarayanan NV, Swanson-Mungerson M, Desai UR. Discovering small-molecule therapeutics against SARSCoV-2. Drug Discov Today. 2020;25(8):1535-1544. doi:10.1016/j. drudis.2020.06.017

13. Russo V, Cardillo G, Viggiano GV, et al. Fondaparinux use in patients with COVID-19: a preliminary multicenter real-world experience. $J$ Cardiovasc Pharmacol. 2020;76(4):369-371. doi:10.1097/ FJC.0000000000000893

14. Russo V, Cardillo G, Viggiano GV, et al. Thromboprophylaxis with fondaparinux versus enoxaparin in hospitalized covid-19 patients: a multicenter Italian observational study. Front Med (Lausanne). 2020;7. doi:10.3389/fmed.2020.569567

15. Pesavento R, Ceccato D, Pasquetto G, et al. The hazard of (sub) therapeutic doses of anticoagulants in non-critically ill patients with Covid-19: the Padua province experience. J Thromb Haemost. 2020;18(10):2629-2635. doi:10.1111/jth.15022

16. Raith EP, Udy AA, Bailey M, et al. Prognostic accuracy of the SOFA score, SIRS criteria, and qSOFA score for in-hospital mortality among adults with suspected infection admitted to the intensive care unit.. JAMA. 2017;317(3):290-300. doi:10.1001/jama.2016.20328

17. Streiff MB, Agnelli G, Connors JM, et al. Guidance for the treatment of deep vein thrombosis and pulmonary embolism. J Thromb Thrombolysis. 2016;41(1):32-67. doi:10.1007/s11239-015-1317-0
18. Lobo JL, Zorrilla V, Aizpuru F, et al. RIETE investigators. D-dimer levels and 15-day outcome in acute pulmonary embolism. Findings from the RIETE registry. J Thromb Haemost. 2009;7(11):1795-1801. doi:10.1111/j.1538-7836.2009.03576.x

19. Schutte T, Thijs A, Smulders YM. Never ignore extremely elevated D-dimer levels: they are specific for serious illness. Neth $\mathrm{J} \mathrm{Med.}$ 2016;74(10):443-448.

20. Di Micco P, D'Uva M, Strina I, et al. The role of D-dimer as first marker of thrombophilia in women affected by sterility: implications in pathophysiology and diagnosis of thrombophilia induced sterility. $J$ Transl Med. 2004;2(1):38. doi:10.1186/1479-5876-2-38

21. Zhou F, Yu T, Du R, et al. Clinical course and risk factors for mortality of adult inpatients with COVID-19 in Wuhan, China: a retrospective cohort study. Lancet. 2020;395(10233):1340. doi:10.1016/S0140-6736(20)30869-2

22. Herold T, Jurinovic V, Arnreich C, et al. Elevated levels of IL-6 and CRP predict the need for mechanical ventilation in COVID-19. $J$ Allergy Clin Immunol. 2020;146(1):128-136.e4. doi:10.1016/j. jaci.2020.05.008

23. Beinrohr L, Murray-Rust TA, Dyksterhuis L, et al. Serpins and the complement system. Methods Enzymol. 2011;499:55-75.

24. Vicci H, Eblen-Zajjur A, López M, Crespo G, Navarro M. Enoxaparin pretreatment effect on local and systemic inflammation biomarkers in the animal burn model. Inflammopharmacology. 2019;27(3):521-529. doi:10.1007/s10787-018-0444-6

25. Shi C, Wang C, Wang H, et al. The potential of low molecular weight heparin to mitigate cytokine storm in severe COVID-19 patients: a retrospective cohort study [published online ahead of print, $2020 \mathrm{Sep}$ 3]. Clin Transl Sci. 2020;13(6):1087-1095. doi:10.1111/cts.12880

26. Marietta M, Vandelli P, Mighali P, et al. Randomised controlled trial comparing efficacy and safety of high versus low low-molecular weight heparin dosages in hospitalized patients with severe COVID-19 pneumonia and coagulopathy not requiring invasive mechanical ventilation (COVID-19 HD): a structured summary of a study protocol. Trials. 2020;21(1):574.

27. Paschoa AF. Heparin: 100 years of pleiotropic effects. J Thromb Thrombolysis. 2016;41(4):636-643. doi:10.1007/s11239-015-1261-z

28. Keshari RS, Silasi R, Popescu NI, et al. Fondaparinux pentasaccharide reduces sepsis coagulopathy and promotes survival in the baboon model of Escherichia coli sepsis. J Thromb Haemost. 2020;18 (1):180-190. doi:10.1111/jth.14642

29. Iba T, Okamoto K, Ohike T, et al. Enoxaparin and fondaparinux attenuates endothelial damage in endotoxemic rats. J Trauma Acute Care Surg. 2012;72(1):177-182. doi:10.1097/TA.0b013e31821a83f0

30. Tan KT, Lip GY. Fondaparinux. Curr Pharm Des. 2005;11(4):415419. doi:10.2174/1381612053382089

31. Di Micco P, Russo V, Lodigiani C. Venous thromboembolism and its association with COVID-19: still an open debate. Medicina (Kaunas). 2020;56(10):506. doi:10.3390/medicina56100506

\section{Publish your work in this journal}

The Journal of Blood Medicine is an international, peer-reviewed, open access, online journal publishing laboratory, experimental and clinical aspects of all aspect pertaining to blood based medicine including but not limited to: Transfusion Medicine; Blood collection, Donor issues, Transmittable diseases, and Blood banking logistics; Immunohematology; Artificial and alternative blood based therapeutics; Hematology; Biotechnology/nanotechnology of blood related medicine; Legal aspects of blood medicine; Historical perspectives. The manuscript management system is completely online and includes a very quick and fair peer-review system. Visit http://www.dovepress.com/testimonials.php to read real quotes from published authors. 